\title{
Mise en service de l'usine de récupération par pompage de Herdecke (Ruhr)
}

Le premier des trois groupes montés dans l'usine de récupération par pompage installée à Herdecke (Ruhr) par la Société des Usines électriques rhénanes-westphaliennes a été mis en service le 20 janvier. La puissance totale des turbines est de entièrement en bétois et constitue, au point de vue technique, une source d'énergie de pointe.

A partir du château d'eau du bassin supérieur, qui est équipé avec des organes de fermeture modernes et des dispositifs auto-

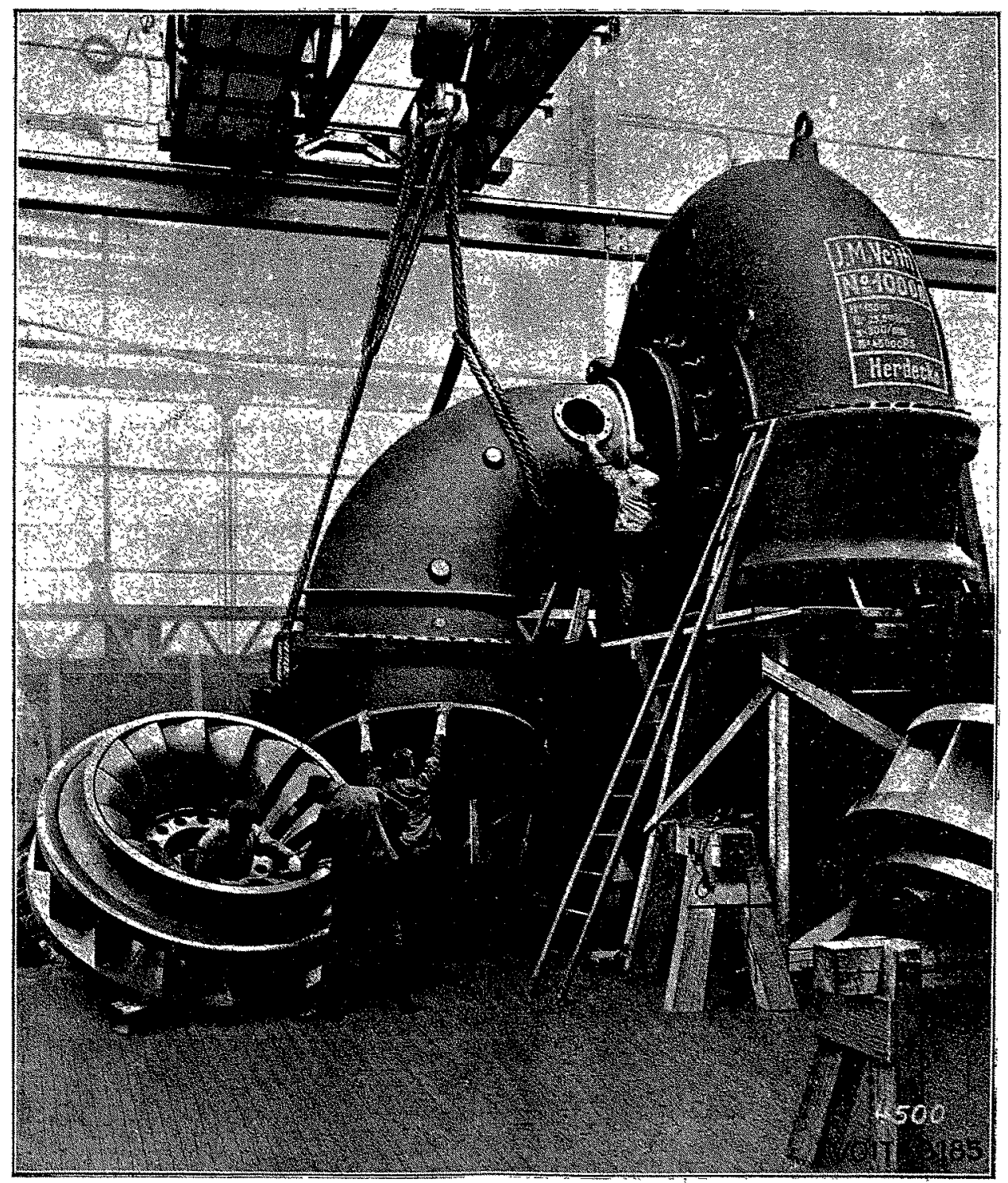

Fig. 1. - Turbine Francis de 48.600 chevaux.

$200.000 \mathrm{CV}$. et celle des pompes de $110.000 \mathrm{CV}$, d'où il résulte que l'usine d'Herdecke dépasse, par ses proportions, toules les installations analogues existantes à ce jour. L'échange d'énergic a lieu, comme toujours, entre deux bassins dont linférieur est formé par un réservoir d'accumulation encadré par la Rulr, Landis que le bassin supérieur "Am Kleff " est artificiel.

Ce dernier, d'une capacité de 1 million de mc. 5, est construit matiques de sécurité, l'eau est amenée aux groupes de machines par quatre conduites forcées indépendantes de $3 \mathrm{~m} .2$ de diamètre

Chaque groupe est composé d'un moteur-générateur, relié rigidement à une turbine, et d'une pompe qui, pendant le service de pompage, est accouplée au moteur-générateur au moyen d'un manchon daccouplement hydro-mécanique pouvant etre embrayé ou débrayé pendant la marche. 


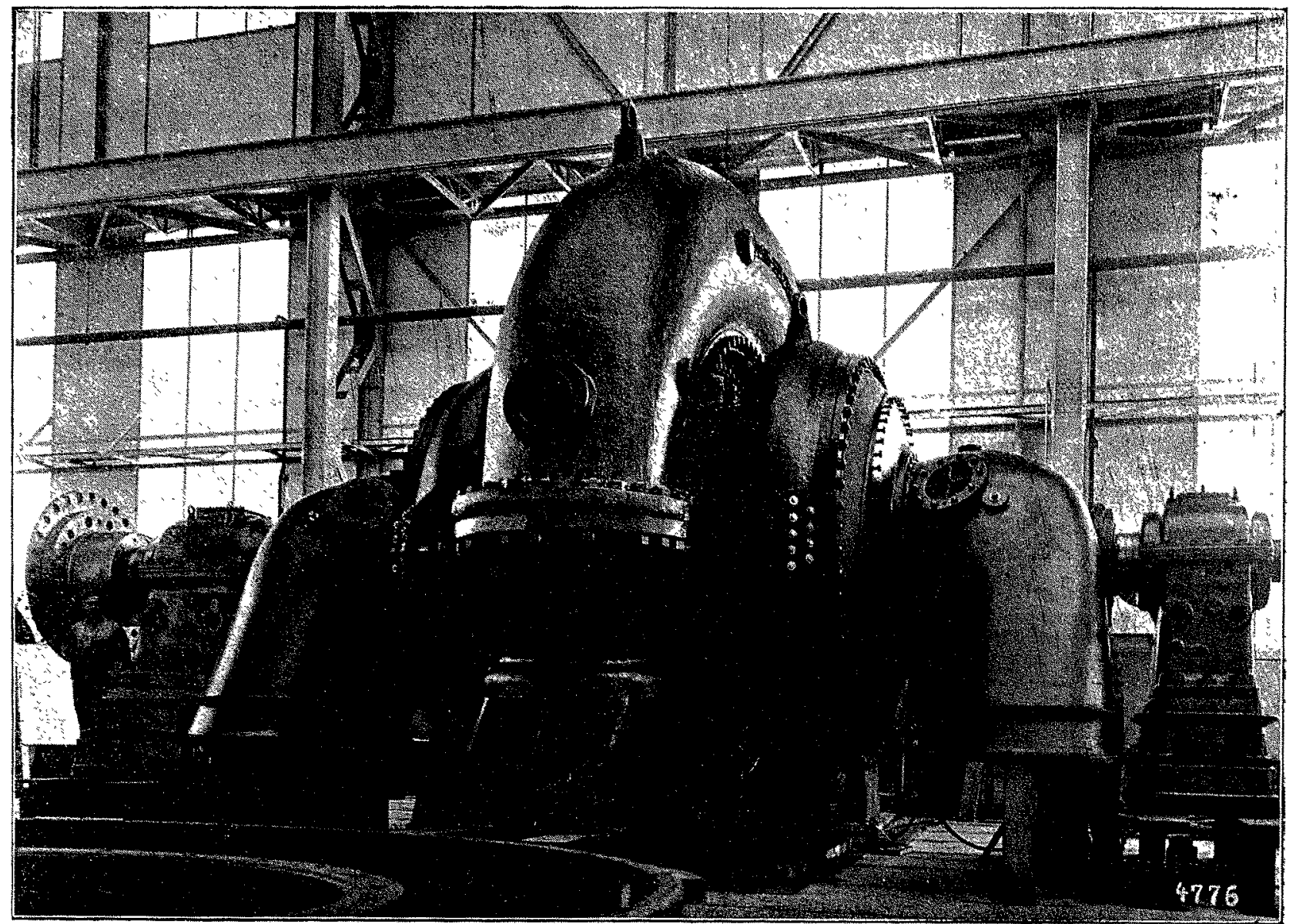

Fig. 2. - Pompe à deux étages absorbant 36.000 chevaux.

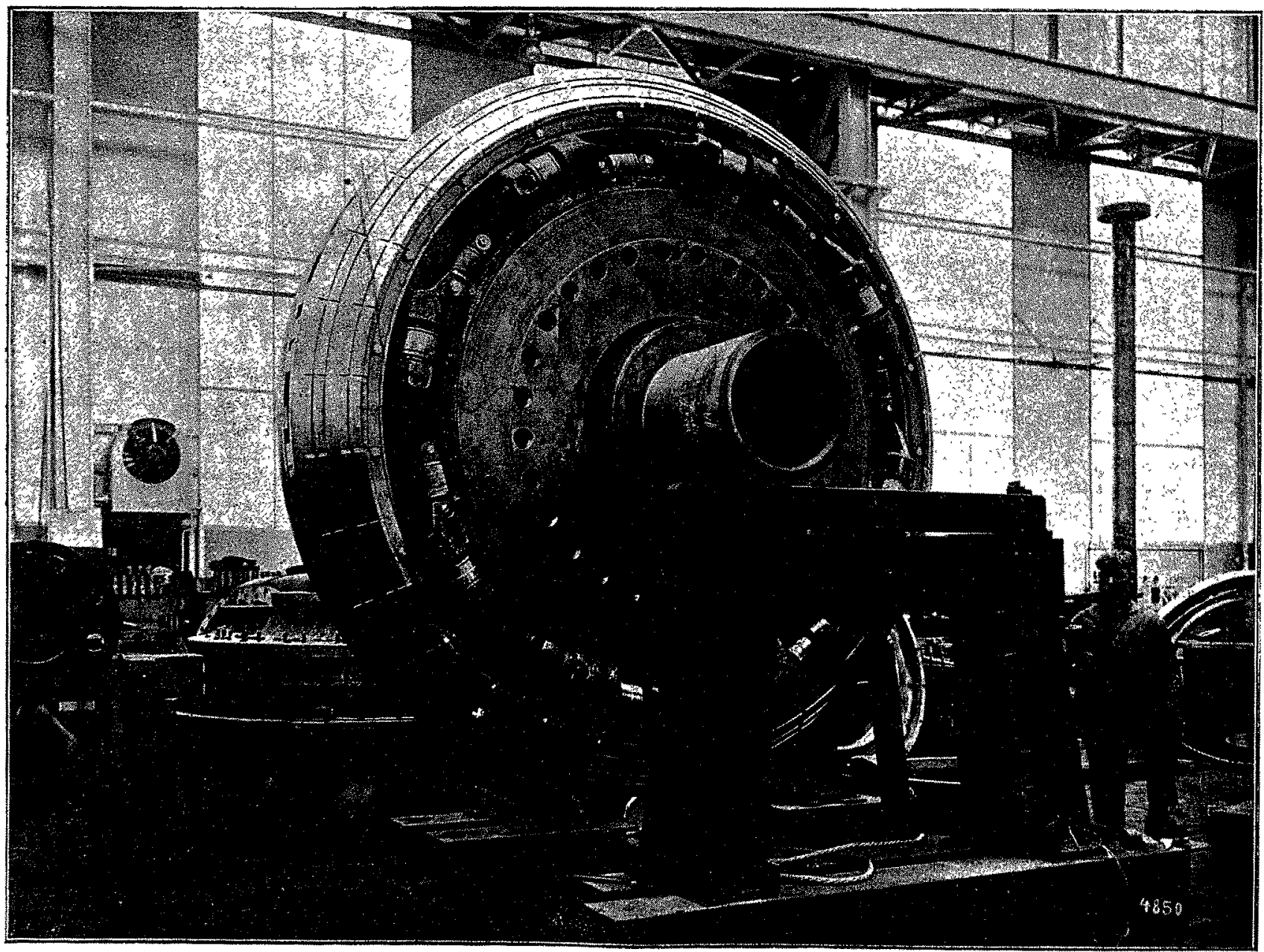

Fig. 3. - Manchon d'accouplement démonté. 
Les turbines du type Francis ayant chacune une puissance normale de $48.600 \mathrm{CV}$. sont les turbines les plus puissantes installées actuellement en Europe (fig. 1).

La construction de ces machines n'a donné lieu à aucune difficulté importante, tandis que celles des pompes et de leurs accouplements n'avait aucun précédent permettant d'en tirer des conclusions de tout repos pour l'établissement de ces grosses unités.

Ceci résultait déjà du fait que la pompe en bâche analogue la plus puissante, existant au moment de la commande, n'absorbail qu'une puissance de $6.400 \mathrm{CV}$., tandis que celle nécessitée pour le refoulement des $15 \mathrm{mc}$./ $/ \mathrm{sec}$. des pompes de "Herdecke " élait de $36.000 \mathrm{CV}$. La plus grande hauteur de refoulement est de $166 \mathrm{~m} . / \mathrm{t}$. 5. Les pompes ont été conçues sur un plan entièrement nouveau par les maisons J.-M. Voith, à Heidenheim et la Société par actions Sulzer Frères, de Ludwigshafen. Elles sont à deux étages et ont deux aspirateurs. Etant donné qu'il étail impossible d'insérer, avec de pareils types de pompes aussi puissantes, des vannes de réglage suffisamment sûres dans le circuit des conduites, il a fallu avoir recours à des appareils diffuseurs à aubes mobiles (fig. 2).

La construction adoptée et brevetée par J.-M. Voith permet d'obtenir, même pour des charges variables, un fonctionnement silencieux de la pompe. Le manchon d'accouplement, système Voith-Fottinger, est également d'une construction nouvelle, remarquable surtout par la grande puissance à transmettre et son fonctionnement absolument exempt de vibrations (fig. 3). Ce résultat est atteint en accélérant la roue de la pompe remplie de la position d'arrêt jusqu'à une vitesse d'environ $95 \%$ de celle de régime au moyen de la partie hydraulique de l'accouplement et d'embrayer ensuite la partie mécanique par l'entremise de laquelle la vitesse normale est atteinte en 35 secondes environ.

Les essais préalables effectués dans les stations d'essai avec des modèles réduits ont permis d'obtenir une mise en service sans encombrement du premier groupe dès l'achèvement de son montage et cela sans aucun accroc quelconque tant au point de vue hydraulique que constructif.

Le groupe est à commande entièrement automatique, de sorte que toutes les mancuvres des turbines et des pompes sont assurées par l'entremise d'un controller à rouleau, situé dans le poste de couplage, transmises ensuite électriquement aux tableaux de commande de la salle des machines et de là, au moyen d'huile sous pression, aux divers servo-moteurs.

Il est possible ainsi, en partant de la position d'arrêt des groupes et de la position fermée des vannes de fermeture, de mettre les turbines en service en 120 secondes, de passér en 150 secondes du service "turbines" au service "pompage ». et du service "pompage au "service "turbines " en 50 secondes environ. C'est la première fois que, pour une installation d'aussi grande puissance, on a appliqué sur une pareille échelle la commande automatique. La mise en service du premier groupe a démontré que cette disposition est réussie en tous points.

Toute la partie hydraulique, turbines, vannes de fermeture et l'appareillage, ainsi que la partie hydraulique de la commande automatique a été livrée par la maison J.-M. Voith d'Heidenheim/Brenz, les pompes ont été livrées en collaboration avec la Société par actions Sulzer Frères, de Ludwigshafen. 